Revista de Matemática: Teoría y Aplicaciones 3(2): 37-48 (1996)

\title{
ANÁLISIS EVOLUTIVO DE LA OPINIÓN EN COSTA RICA: 1988-1991
}

\author{
William Castillo Elizondo ${ }^{1}$ - Jorge GonzÁlez ${ }^{2}$
}

\begin{abstract}
Resumen
En este artículo se utiliza el método Statis para analizar la evolución de la Opinión de los Costarricenses mayores de 18 años. Para tal efecto se usaron las encuestas anuales de opinión realizadas en el período 1988-1991, específicamente en aquellas preguntas que se repitieron en los cuatro años [9]
\end{abstract}

Palabras clave: método Statis, intraestructura, interestructura, compromiso, tablas múltiples.

\section{Introducción}

El análisis de los fenómenos sociales o de otra naturaleza, puede ser abordado desde el ángulo de su evolución o de una manera estática. Así nuestro enfoque en relación con el fenómeno de la opinión es descubrir sus tendencias evolutivas e intentar explicarlas.

Técnicamente este tipo de estudios se denomina análisis de tablas múltiples y existen varios métodos para su implementación; los cuales marcan su principal diferencia en la forma como se elige el operador compromiso. En el presente caso se ha utilizado el método STATIS (Estadística de tres índices), del cual se ofrece enseguida una breve descripción, enmarcada en las condiciones del caso que nos ocupa. (Una presentación más amplia se encuentra en [2], [3], [7], [6], [5], [8]).

\section{$2 \quad$ El método Statis}

EL Método Statis es una metodología estadístico-matemática utilizada para hacer el análisis descriptivo de varias tablas de datos. Este método fue concebido y desarrollado exclusivamente para el análisis de tablas de datos del tipo individuos $\times$ variables cuantitativas, muy frecuentemente, como en este caso, observadas durante un período determinado. En general suponemos que se tienen $K$ matrices de tamaño $n \times p_{i}$, de observaciones de

\footnotetext{
${ }^{1}$ Programa de Investigación en Modelos y Análisis de Datos (PIMAD), Escuela de Matemática, Universidad De Costa RicA, 2060 San José, Costa Rica. E-Mail: wcastill@cariari.ucr.ac.cr

${ }^{2}$ Misma dirección postal. E-Mail: jgonzale@cariari.ucr.ac.cr
} 
$p_{i}$ variables (conflictos) sobre $n$ individuos los cuales, en este estudio, son los centros de gravedad con pesos iguales. Este método consta de tres fases importantes, a saber : la Intraestructura, el Compromiso y la Interestructura.

\subsection{La interestructura}

$\mathrm{Su}$ objetivo es comparar globalmente la estructura de las diferentes tablas de datos, lo cual se realiza a través del estudio de las matrices $W_{i}=X_{i} X_{i}^{t}$ de productos escalares entre individuos. Para obtener una descripción de estas $K$ matrices usamos la métrica definida por el producto interno.

$$
<W_{i}, W_{j}>_{\Phi}=\operatorname{tr}\left(W_{i} W_{j}\right)
$$

Una representación plana de la nube $\left\{W_{1}, \ldots, W_{K}\right\}$ se obtiene diagonalizando la matriz $S$ definida como $S_{i j}=<W_{i}, W_{j}>_{\Phi}$ y se proyectan los $W_{i}, \Phi$-ortogonalmente sobre los ejes determinados por los vectores propios de $S$. Esto es equivalente a realizar un ACP sobre el triplete $\left(X, I_{r}, \frac{1}{n^{2}} I\right)$, donde $X$ es la matriz cuyas columnas son los operadores $W_{i}$ escritos como vectores de $\mathbb{R}^{n^{2}}$. Así, la representación bidimensional de los operadores es la representación de las variables de este ACP, en el círculo de correlaciones.

\subsection{El compromiso}

En análisis de tablas múltiples, los inidviduos y las variables de tablas distintas, pertenecen a espacios de dimensión diferente. Esta circunstancia obliga a determinar un operador promedio llamado compromiso. Los ejes determinados por los vectores propios de este operador constituyen un sistema ortonormado en el cual se pueden representar todos los individuos y todas las variables. Existen varias definiciones del compromiso, una de las cuales es la de H. L'Hermier des Plantes que consiste en elegir como compromiso la primera componente principal $W=\sum \alpha_{i} W_{i}$ del ACP anterior. Esta definición es posible gracias a que todas las entradas de la matriz $S$ son positivas de modo que, por el Teorema de Frobenius ([1]), $\alpha_{1}, \ldots, \alpha_{K}$ pueden elegirse positivos.

\subsection{La intraestructura}

El objetivo de esta fase es buscar las causas de la diferenciación observada a partir de la interestructura. El procedimiento consiste en proyectar las variables y los individuos sobre sus respectivos ejes del compromiso. Para lo cual se debe considerar la matriz de tamaño $\left(n \times \sum p_{i}\right)$,

$$
X=\left(\sqrt{\alpha_{1}} X_{1}, \ldots, \sqrt{\alpha_{K}} X_{K}\right)
$$

El compromiso es precisamente $X X^{t}$. El ACP del triplete $(X, I, D)$ nos da una representación simultánea de las $\sum p_{i}$ variables en la base $D$-ortonormal de las componentes principales, donde $D$ es la métrica de pesos.

Para representar los individuos usamos la técnica de elemento suplementario. Así el i-ésimo individuo del k-ésimo año es la fila i-ésima de la matriz $W_{k} D C$, donde $\mathrm{C}$ es la matriz de las componentes principales normalizadas. 


\section{Los datos}

Los datos de base de esta investigación corresponden a las encuestas de opinión de los años 1988, 1989,1990 y 1991 realizadas por el proyecto de investigación Estructuras de la Opinión Pública. Estas encuestas son anuales sobre una muestra de 1000 individuos, mayores de 18 años, preservando cuotas por sexo, edad y professión. Los entrevistados se pronuncian sobre unas 100 afirmaciones relativas a los principales conflictos sociales ventilados por la prensa local [9].

Un ejemplo de tales afirmaciones es:

\section{La democracia es el sufragio}

Los entrevistados deben responder una de las siguientes alternativas:

- En total desacuerdo

- Bastante en desacuerdo

- Podría estar de acuerdo

- Bastante de acuerdo

- Totalmente de acuerdo

- No responde

Estas respuestas se codifican en una escala de 1 a 6 .

\section{Selección de las variables}

Nos limitaremos a hacer un análisis evolutivo de la opinión, teniendo en cuenta únicamente aquellas preguntas que se repiten en todas las encuestas.

Así entonces, decidimos escoger las siguientes variables que son las que permanecieron en el periodo 88-91:

- El Seguro social debe privatizarse (SP).

- Se debe propiciar la libre elección médica (LM).

- La democracia es el sufragio (DS).

- Se debe defender la banca nacionalizada (BN).

- Se debe confiar en la justicia (CJ).

- Se debe aumentar el presupuesto en seguridad (PS).

- Ya no nos sentimos seguros (IS).

- Los enfermos de sida deben aislarse (SD). 


\section{Construcción de la tabla de datos}

Para analizar la evolución de la opinión hemos considerado la opinión promedio de los grupos sociales determinados por las siguientes variables:

- Provincia: San José (Sjos), Alajuela (Ala), Cartago (Car), Guanacaste (Gua), Puntarenas (Aren), Limón (Lim) y Heredia (Her).

- Edad, codificada en las siguientes clases: de 18 a 25 (A18), de 25 a 35 (A25), de 35 a 45 (A35), de 45 a 55 (A45) y más de 55 (A 55 ).

- Sexo: Hombres (H) y Mujeres (M).

- Escolaridad: Ninguna (N), Primaria (P), Secundaria (S), Técnica (T), Universitaria incompleta (UI) y Universitaria completa (UC).

- Profesión: Agricultor (Bag), Patrón o empleado de comercio (Bec), Profesión liberal (Bpl), Empleado (Bem), Profesión intermedia (Bpi), maestro-profesor (Bmp), pensionado (Bpen), Ama de casa (Bac) y Desempleado (Bdes)

- Partido político: Partido Liberación Nacional (PLN), Partido Unidad Social Cristiana (PUSC), Izquierda (Izq), Otros partidos (Potr) y Ningún partido (Pnin).

- Ingreso Familiar: Ingresos Bajos (IB), Ingresos Medios (IM) e Ingresos Altos (IA).

La tabla de datos se construye usando los centros de gravedad como filas (individuos), para obtener así una matriz de tamaño $42 \times 8$ por cada año.

\section{Resultados}

Utilizando el sistema PIMAD-STATIS [4] hemos obtenido los siguientes resultados : 


\subsection{Análisis de la interestructura}

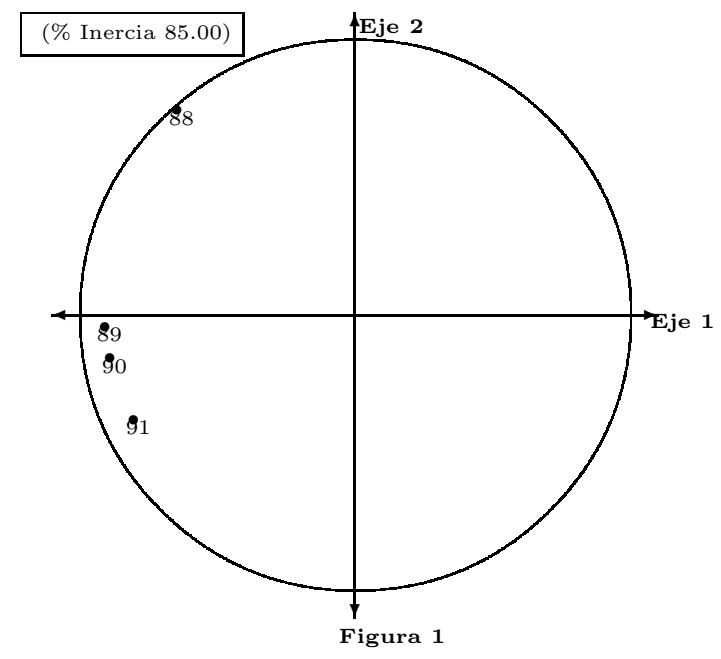

Para hacer una representación plana de la distribución espacial de las opiniones correspondientes a cada uno de los cuatro años, calculamos una imagen euclídea aproximada de los operadores $W D$, lo que se obtiene considerando el primer plano principal del ACP sobre la matriz cuyas variables son los $W_{i}$.

La Figura 1 muestra la evolución global de la opinión anual en el periodo 1988-1991 en el primer plano principal el cual explica un $85 \%$ de la inercia total. Los puntos representativos de cada encuesta se encuentran muy próximos a la circunferencia de radio uno, lo que garantiza una buena representación de la realidad descrita por las variables tomadas en cuenta en este estudio.

Se observa una estabilidad de la opinión en los años 89, 90, 91 diferenciándose de la estructura de la opinión en 1988. Esta diferenciación la analizaremos en el contexto de la Intraestructura.

La configuración espacial de los individuos representada por los operadores $W_{k}$, se resume en el operador compromiso $W$ que no es otra cosa que la primera componente principal del ACP mencionado en la sección (2.1). En la Figura1 se observa que este operador representa bien la estructura común de la opinión de los años 89-90-91. Como puede comprobarse en las Tablas de Correlaciones (Ver Apéndice), la proximidad en estos mismos años es coherente con la estabilidad observada en las matrices de correlaciones correspondientes y, por otra parte, con las ortogonalidades mayoritarias entre las variables de estos años con el año 1988.

\subsection{Análisis de la intraestructura}

El análisis de la intraestructura consiste en hacer representaciones planas de las variables y de los individuos en los ejes del operador compromiso. Un análisis en componentes principales sobre la tabla que se obtiene concatenando las tablas de los centros de gravedad de cada año y ponderándolas adecuadamente, nos permite obtener tales representaciones. 
La Figura 2 corresponde a la representación de las variables en los dos primeros ejes los cuales explican el $58 \%$ de la inercia total.

Se observa que las variables SD, DS, SP y en menor grado BN y CJ, correlacionan con el primer eje. Por otra parte las variables LM e IS lo hacen con el segundo eje. En el caso de las variables BN y CJ se observa una evolución (movimiento) sobre el primer eje que es explicada en parte por la diferenciación del año 89 con respecto a los restantes.

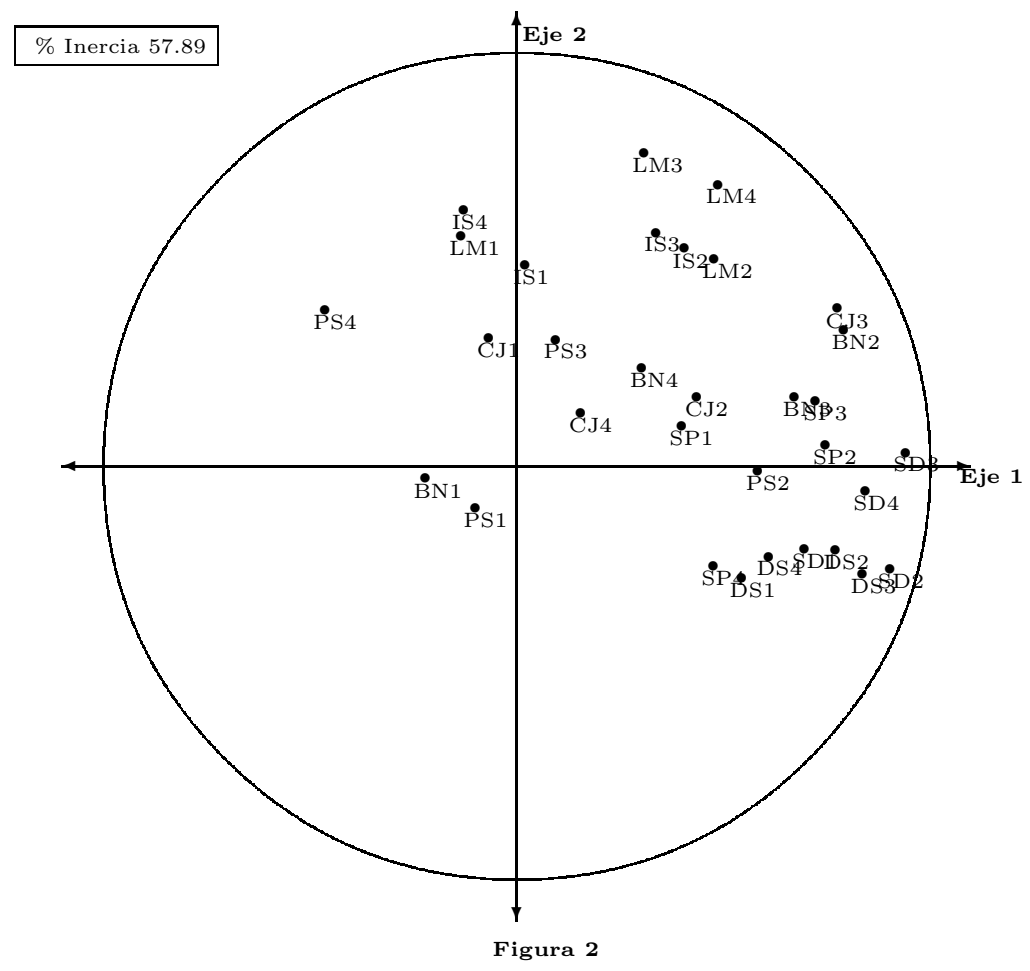




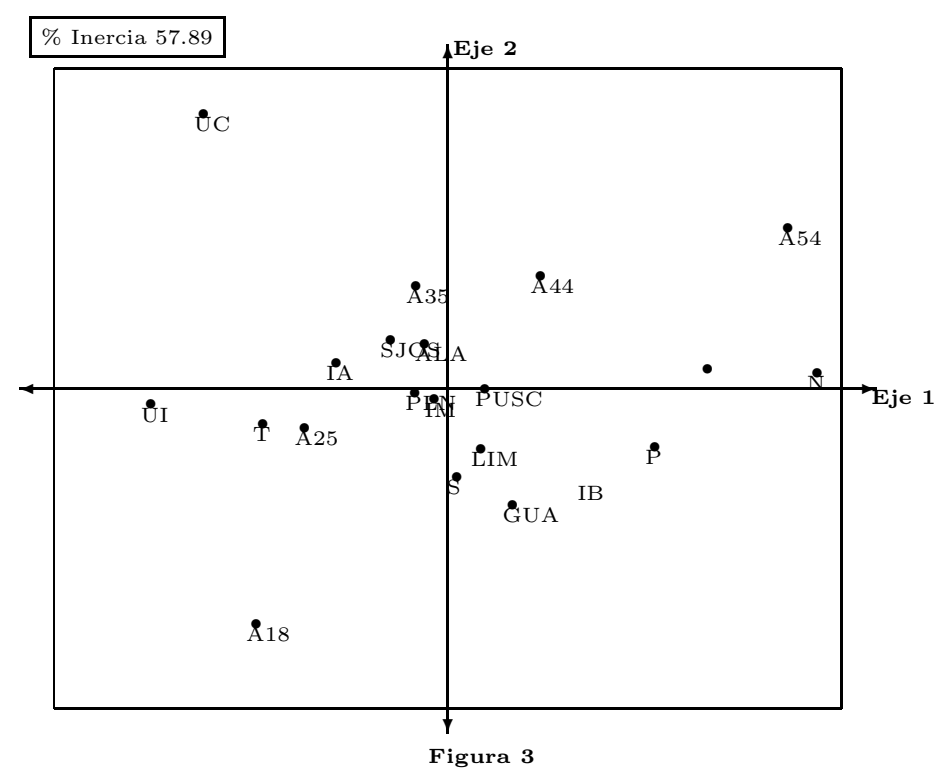

En la Figura 3 se representan los grupos sociales promedio en los ejes del compromiso, y en las Figuras 5 y 6 las trayectorias.

Se nota que el nivel de escolaridad es un factor claramente discriminante en cuanto a la opinión. Los grupos de mayor escolaridad UC arriba en el segundo cuadrante se oponen a los grupos sociales de menor escolaridda (P) que son a su vez los de más bajos ingresos (IB). Asociadas con estos grupos se encuentran, respectivamente, las provincias de mayor desarrollo como San José y Alajuela, en oposición a Limón y Guanacaste. Las diferencias de opinión más claras derivan de las variables que determinan en mayor grado esas direcciones. Es así como a partir de las observaciones anteriores complementadas con los histogramas (Ver Apéndice), podemos decir lo siguiente:

- En los grupos sociales de más alta escolaridad como EUC se nota una evolución en el sentido de disminuir su confianza en la justicia, aumentar su deseo de que se invierta más presupuesto en seguridad y cada año el sentimento de inseguridad es mayor. Respecto a la enfermedad del sida estos grupos sociales son cada vez más tolerantes. En los sectores de menos escolaridad como EP y EN esta tendencia es menos pronunciada y más irregular (Ver Histogramas).

- Hay una diferenciación de la opinión entre los grupos de menor edad con respecto a los de mayor edad, la cual se expresa en grado creciente de desconfianza en la justicia de parte de los jóvenes que ha su vez son más tolerantes respecto al sida, que las personas de mayor edad (Ver Figuras: 5, 6).

- En relación con los grupos de filiación política, hemos constatado que la diferenciación proviene de los grupos minoritarios fuera del PLN y del PUSC. Estos dos últimos partidos son indistinguibles desde el ángulo de la opinión respecto de las 
variables analizadas, como lo ponen en evidencia las representaciones en la Figura 4. Este hecho también lo hemos verificado con un segundo análisis donde los partidos minoritarios (Izquierda, Otros y Ninguno) no fueron tomados en cuenta.

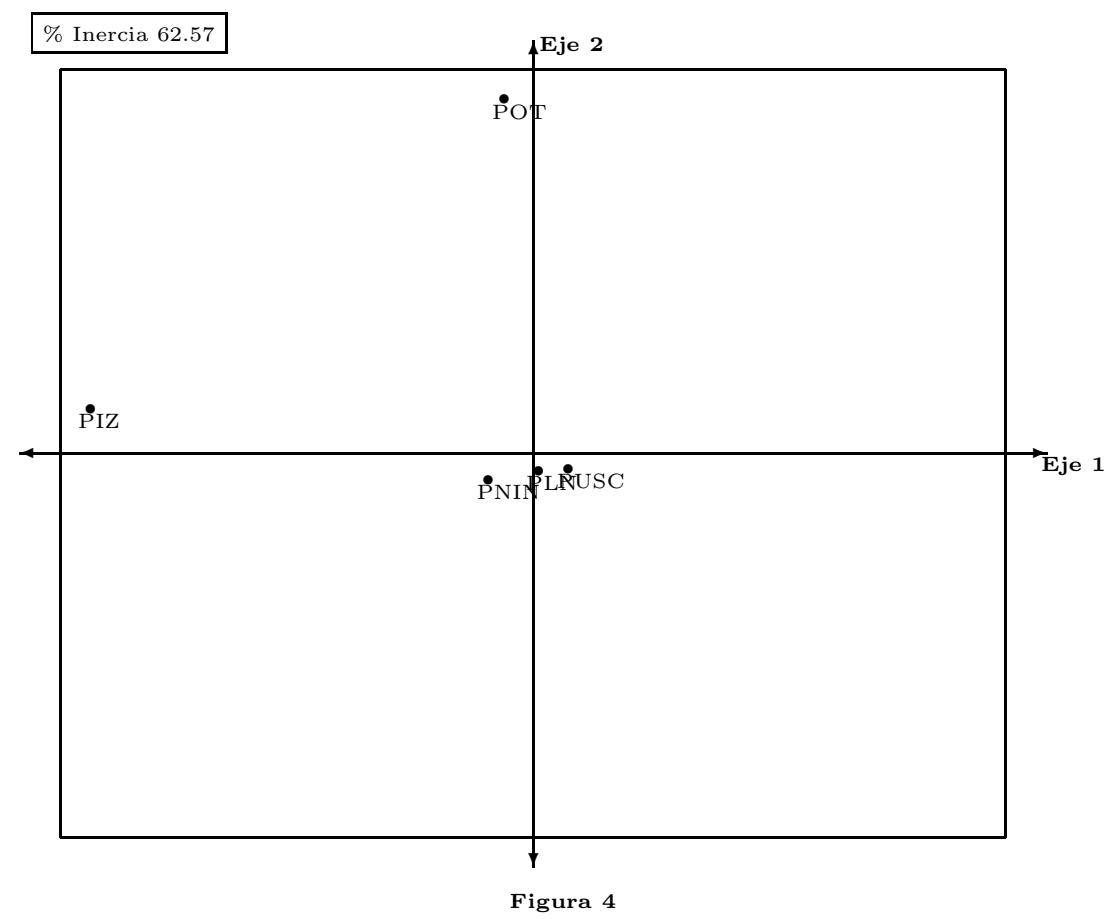

\section{Conclusiones y perspectivas}

De acuerdo con los análisis realizados podemos señalar lo siguiente:

1. La opinión en el año 1988 se diferencia de los tres años siguientes. A efecto de poder explicar esta situación es necesario superar ciertas limitaciones como son, por ejemplo, la necesidad de incluir en los análisis más variables y abordar la fase de interpretación con participación multidisciplinaria, especialmente con cientistas sociales.

2. Pese a las limitaciones señaladas en el punto anterior, se puede afirmar que el movimiento evolutivo de las variables Defender la banca nacionalizada, Aumentar el presupuesto en seguridad y Se puede confiar en la justicia; marcan una clara diferencia de la opinión en 1988 con respecto a la opinión en los otros años.

3. Los resultados obtenidos confirman que la edad y el nivel de escolaridad son variables diferenciadoras de la opinión. Mientras tanto la filiación política no introduce diferencias de opinión importantes, con excepcion de los grupos minoritarios. Desde este 
ángulo, queda justificada la apreciación en el sentido que los partidos tradicionalmente mayoritarios desde hace algunos años representan esencialmente lo mismo. Esto es así, aun cuando 5 años atrás, durante el período en que se realizaron estas encuestas, la mayor parte de los ciudadanos no lo percibían claramente. En esta perspectiva cabe preguntarse si actualmente los ciudadanos son conscientes de lo que representan el PLN y el PUSC y si perciben diferencias importantes entre estos dos partidos. Para argumentar una respuesta a estas dos preguntas es necesario extender el período de análisis a más años y considerar suficientes conflictos.

4. Dentro de las actividades futuras de investigación ocupan un lugar prefencial el desarrollo de Sofware y la consolidación del sistema PIMAD, con el fin de que se puedan considerar variables diferentes año a año y hacer un estudio evolutivo en períodos más extensos. Esto nos permitiría constatar la permanencia de tendencias, su desaparición o modificación y el surgimiento de otras. Por otra parte el análisis se enriquece con la posibilidad de considerar, de una manera más completa, la variedad de conflictos sociales que intervienen en la formación de la opinión.

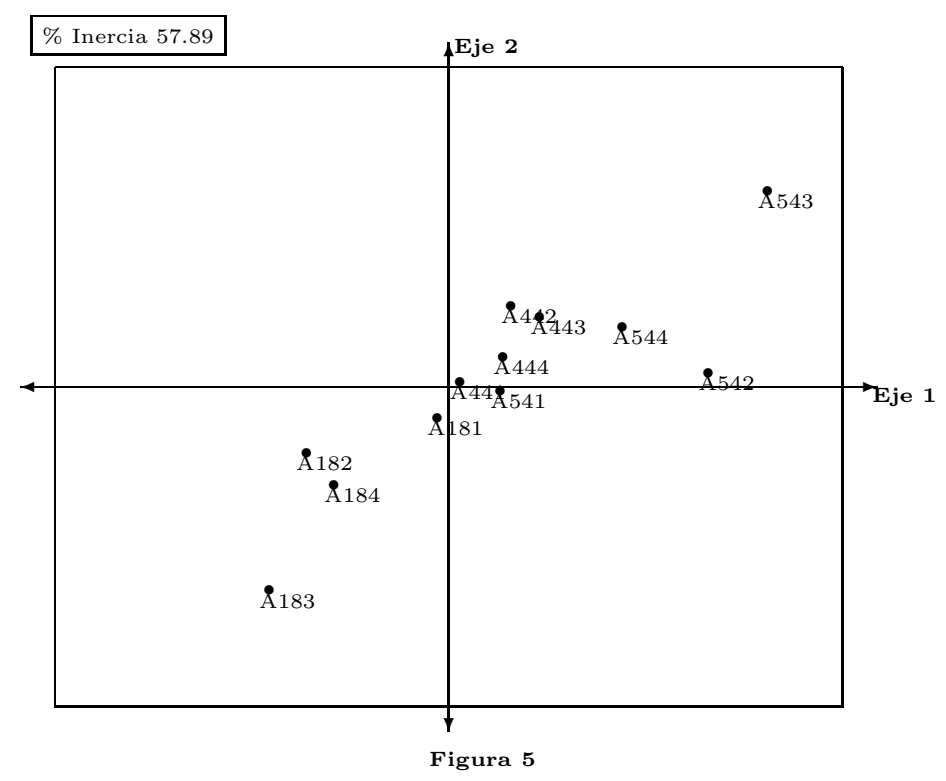




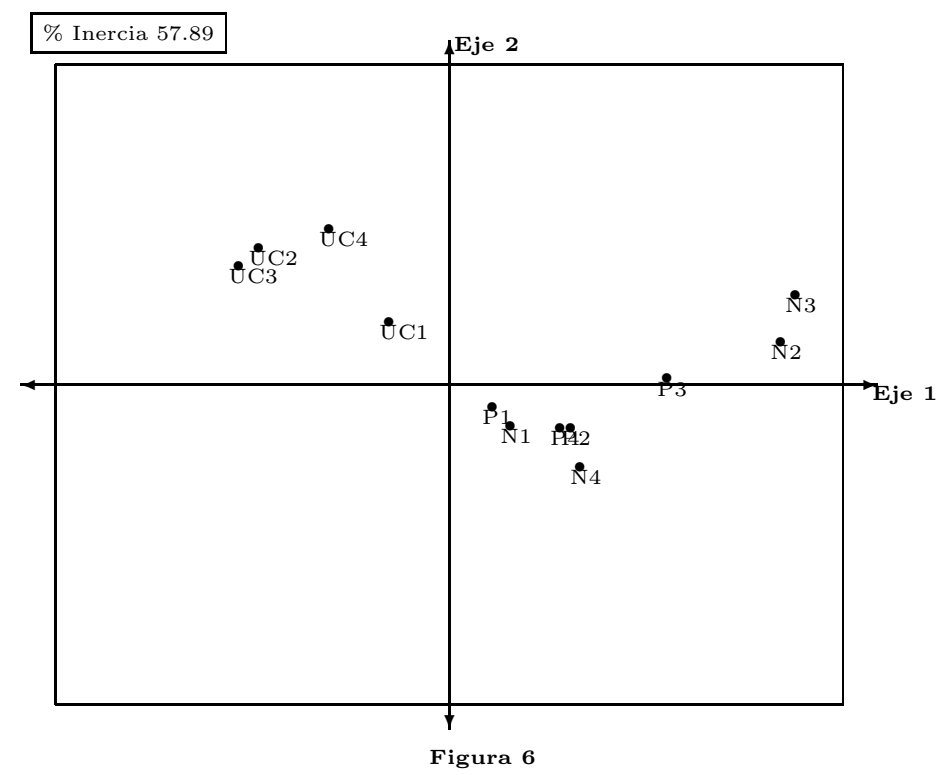

\section{Referencias}

[1] Acuña, O.; Ulate, F. (1994) Matrices no negativas. Editorial de la Universidad de Costa Rica, San José.

[2] Castillo, W.; González, J. (1994) "Análisis de tablas múltiples de datos". Rev. de Matemática: Teoría y Aplicaciones, 1(1): 47-55.

[3] Glaçon, F. (1981) Analyse Conjointe de Plusieurs Matrices de Données. Thèse de 3ème cycle, Université Scientifique et Médicale de Grenoble.

[4] González, J.; Rodríguez, O. (1995) "Algoritmo e implementación del método Statis". IX Simposio Métodos Matemáticos Aplicados a las Ciencias, J. Trejos (ed.), Turrialba: $49-56$.

[5] González, J. (1994) "Estudio evolutivo de la calidad del agua", Memoria del II Encuentro Centroamericano de Investigadores en Matemáticas, G. Mora (ed.), San Ramón: 425-434.

[6] Lavit, Ch. (1988) Analyse Conjointe de Tableaux Quantitatifs. Masson, Paris.

[7] Chevalier, F. (1990) "L'analyse en composantes conjointes d'une famille de triplets indexés", Statistique et Analyse des Données, Vol 2: 35-75.

[8] L'Hermier-des-Plantes, H. (1976) "Structuration des Tableaux à Trois Indices de la Statistique", Thèse de 3ème cycle, Université de Montpellier II. 
[9] Poltronieri, J. (1989-1991) "Estructuras de la Opinión Pública en Costa Rica". Informes de Investigación del período 1989-1991. Escuela de Matemáticas, Universidad de Costa Rica. 


\section{APENDICE}

Histogramas de las respuestas promedio a las preguntas: Se debe confiar en la justicia (CJ), Se debe aumentar el presupuesto en seguridad (PS), Ya no nos sentimos seguros (IS), Los enfermos de sida deben aislarse (SD), dadas por los grupos sociales ordenados según: Grupos de edad, nivel educativo, nivel de ingreso y filiación política

\begin{tabular}{|c|c|c|c|c|}
\hline CG & CJ & $\overline{\mathrm{PS}}$ & IS & SD \\
\hline A 18 & 3.153 & 3.676 & 3.610 & 3.554 \\
\hline A 18 & 2.679 & 3.879 & 3.555 & 3.386 \\
\hline A18 & 2.504 & 3.879 & 3.613 & 2.603 \\
\hline A18 & 2.510 & 3.977 & 3.875 & 2.449 \\
\hline A54 & 3.292 & 3.746 & 4.031 & 3.950 \\
\hline A 54 & 3.000 & 3.977 & 4.034 & 4.022 \\
\hline A54 & 3.125 & 4.121 & 4.136 & 3.547 \\
\hline A54 & 2.734 & 4.037 & 4.232 & 3.419 \\
\hline EN & 3.200 & 3.242 & 3.769 & 3.969 \\
\hline EN & 3.167 & 3.902 & 4.220 & 4.063 \\
\hline EN & 3.245 & 3.739 & 3.918 & 3.438 \\
\hline EN & 2.816 & 3.816 & 3.974 & 3.263 \\
\hline EP & 3.265 & 3.729 & 3.800 & 3.876 \\
\hline EP & 2.807 & 3.949 & 3.800 & 3.881 \\
\hline EP & 2.812 & 4.067 & 3.935 & 3.431 \\
\hline EP & 2.531 & 3.960 & 3.906 & 3.162 \\
\hline EUC & 3.319 & 3.602 & 3.836 & 3.381 \\
\hline EUC & 2.837 & 3.724 & 3.871 & 3.035 \\
\hline EUC & 2.663 & 3.971 & 4.049 & 2.481 \\
\hline EUC & 2.532 & 4.063 & 4.351 & 2.425 \\
\hline EUI & 3.266 & 3.699 & 3.788 & 3.475 \\
\hline EUI & 2.698 & 3.860 & 3.569 & 2.965 \\
\hline EUI & 2.538 & 3.938 & 3.793 & 2.406 \\
\hline EUI & 2.527 & 4.086 & 4.122 & 2.242 \\
\hline IA & 3.191 & 3.736 & 3.866 & 3.614 \\
\hline IA & 2.750 & 3.841 & 3.708 & 3.402 \\
\hline IA & 2.648 & 4.070 & 3.885 & 2.666 \\
\hline IA & 2.548 & 4.111 & 4.141 & 2.582 \\
\hline IB & 3.199 & 3.649 & 3.749 & 3.701 \\
\hline IB & 2.786 & 3.943 & 3.887 & 3.708 \\
\hline IB & 2.617 & 3.902 & 3.789 & 3.336 \\
\hline IB & 2.591 & 3.834 & 4.036 & 3.109 \\
\hline IM & 3.258 & 3.806 & 3.925 & 3.761 \\
\hline IM & 2.733 & 3.959 & 3.802 & 3.498 \\
\hline IM & 2.696 & 4.008 & 3.940 & 2.945 \\
\hline IM & 2.411 & 3.958 & 3.958 & 2.783 \\
\hline
\end{tabular}




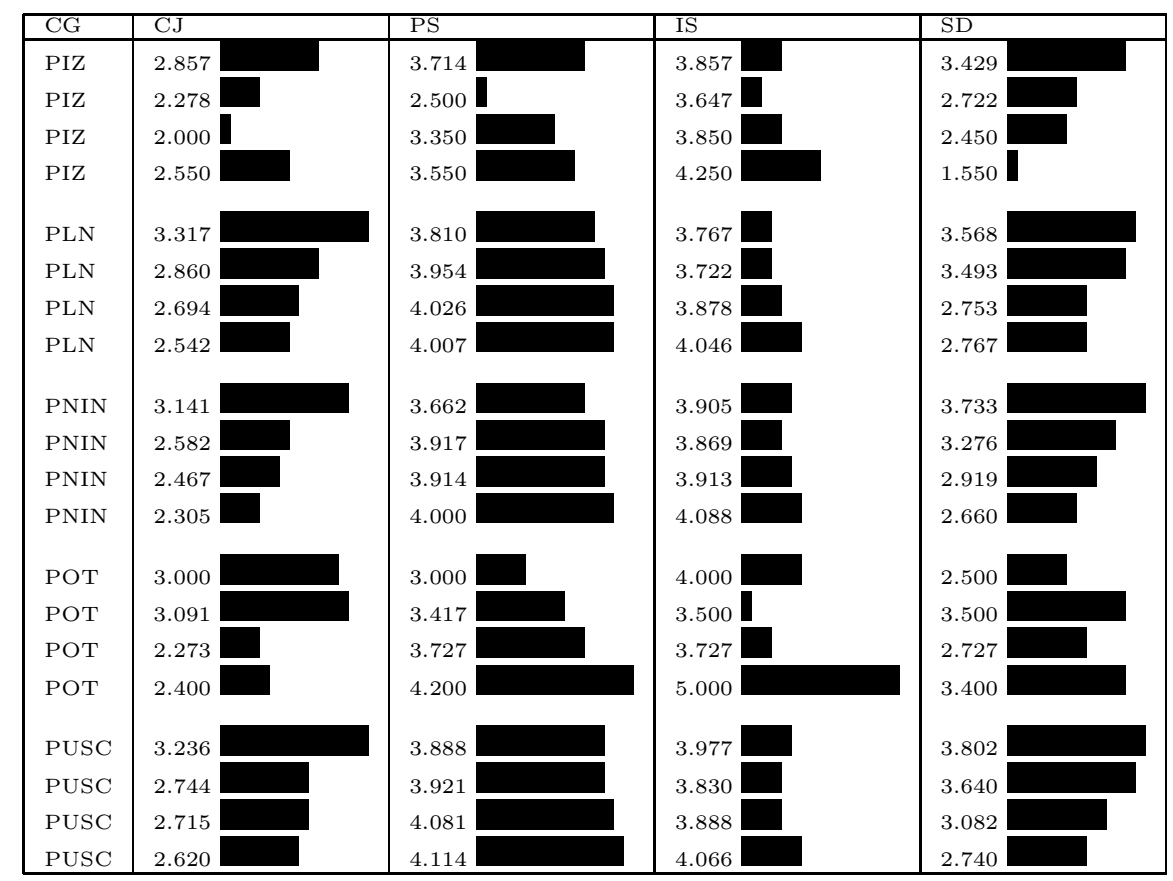

Correlaciones entre las variables del año 1989

\begin{tabular}{|c|c|c|c|c|c|c|c|c|}
\hline & SP1 & LM1 & DS1 & BN1 & CJ1 & PS1 & IS1 & SD1 \\
\hline SP1 & 1.00 & 0.01 & 0.27 & -0.26 & 0.11 & -0.18 & 0.29 & 0.47 \\
LM1 & 0.01 & 1.00 & -0.01 & 0.31 & 0.27 & 0.27 & 0.51 & -0.32 \\
DS1 & 0.27 & -0.01 & 1.00 & 0.07 & -0.03 & 0.29 & 0.15 & 0.45 \\
BN1 & -0.26 & 0.31 & 0.07 & 1.00 & 0.43 & 0.40 & 0.01 & -0.11 \\
CJ1 & 0.11 & 0.27 & -0.03 & 0.43 & 1.00 & -0.08 & 0.03 & -0.27 \\
PS1 & -0.18 & 0.27 & 0.29 & 0.40 & -0.08 & 1.00 & 0.06 & 0.06 \\
IS1 & 0.29 & 0.51 & 0.15 & 0.01 & 0.03 & 0.06 & 1.00 & -0.03 \\
SD1 & 0.47 & -0.32 & 0.45 & -0.11 & -0.27 & 0.06 & -0.03 & 1.00 \\
\hline
\end{tabular}

Correlaciones entre las variables del año 1990

\begin{tabular}{|c|c|c|c|c|c|c|c|c|}
\hline & SP2 & LM2 & DS2 & BN2 & CJ2 & PS2 & IS2 & SD2 \\
\hline SP2 & 1.00 & 0.13 & 0.41 & 0.47 & 0.31 & 0.48 & 0.19 & 0.71 \\
LM2 & 0.13 & 1.00 & 0.55 & 0.61 & 0.32 & 0.07 & 0.76 & 0.33 \\
DS2 & 0.41 & 0.55 & 1.00 & 0.54 & 0.24 & 0.35 & 0.38 & 0.76 \\
BN2 & 0.47 & 0.61 & 0.54 & 1.00 & 0.45 & 0.48 & 0.60 & 0.66 \\
CJ2 & 0.31 & 0.32 & 0.24 & 0.45 & 1.00 & 0.36 & 0.45 & 0.34 \\
PS2 & 0.48 & 0.07 & 0.35 & 0.48 & 0.36 & 1.00 & 0.17 & 0.50 \\
IS2 & 0.19 & 0.76 & 0.38 & 0.60 & 0.45 & 0.17 & 1.00 & 0.26 \\
SD2 & 0.71 & 0.33 & 0.76 & 0.66 & 0.34 & 0.50 & 0.26 & 1.00 \\
\hline
\end{tabular}


Correlaciones entre las variables del año 1991

\begin{tabular}{|c|c|c|c|c|c|c|c|c|}
\hline & SP3 & LM3 & DS3 & BN3 & CJ3 & PS3 & IS3 & SD3 \\
\hline SP3 & 1.00 & 0.25 & 0.53 & 0.36 & 0.71 & -0.18 & 0.43 & 0.65 \\
LM3 & 0.25 & 1.00 & 0.22 & 0.41 & 0.55 & 0.45 & 0.34 & 0.26 \\
DS3 & 0.53 & 0.22 & 1.00 & 0.45 & 0.52 & 0.05 & 0.05 & 0.81 \\
BN3 & 0.36 & 0.41 & 0.45 & 1.00 & 0.57 & 0.25 & 0.30 & 0.57 \\
CJ3 & 0.71 & 0.55 & 0.52 & 0.57 & 1.00 & 0.01 & 0.37 & 0.77 \\
PS3 & -0.18 & 0.45 & 0.05 & 0.25 & 0.01 & 1.00 & 0.35 & 0.09 \\
IS3 & 0.43 & 0.34 & 0.05 & 0.30 & 0.37 & 0.35 & 1.00 & 0.31 \\
SD3 & 0.65 & 0.26 & 0.81 & 0.57 & 0.77 & 0.09 & 0.31 & 1.00 \\
\hline
\end{tabular}

Correlaciones entre las variables del año 1992

\begin{tabular}{|c|c|c|c|c|c|c|c|c|}
\hline & SP4 & LM4 & DS4 & BN4 & CJ4 & PS4 & IS4 & SD4 \\
\hline SP4 & 1.00 & 0.13 & 0.07 & -0.34 & -0.25 & -0.36 & 0.08 & 0.29 \\
LM4 & 0.13 & 1.00 & 0.12 & 0.41 & -0.08 & 0.04 & 0.46 & 0.32 \\
DS4 & 0.07 & 0.12 & 1.00 & 0.35 & 0.20 & -0.27 & -0.22 & 0.53 \\
BN4 & -0.34 & 0.41 & 0.35 & 1.00 & 0.22 & -0.06 & -0.16 & 0.21 \\
CJ4 & -0.25 & -0.08 & 0.20 & 0.22 & 1.00 & 0.21 & -0.31 & 0.18 \\
PS4 & -0.36 & 0.04 & -0.27 & -0.06 & 0.21 & 1.00 & 0.40 & -0.48 \\
IS4 & 0.08 & 0.46 & -0.22 & -0.16 & -0.31 & 0.40 & 1.00 & -0.25 \\
SD4 & 0.29 & 0.32 & 0.53 & 0.21 & 0.18 & -0.48 & -0.25 & 1.00 \\
\hline
\end{tabular}

Correlaciones entre las variables de los años 1992, 1991, 1990. con las variables del año 1989

\begin{tabular}{|c|c|c|c|c|c|c|c|c|}
\hline & SP1 & LM1 & DS1 & BN1 & CJ1 & PS1 & IS1 & SD1 \\
\hline SSP4 & 0.12 & -.22 & 0.00 & -.28 & -.35 & -.13 & -.19 & 0.33 \\
LM4 & 0.11 & 0.41 & 0.14 & 0.03 & 0.05 & 0.10 & 0.37 & 0.18 \\
DS4 & 0.37 & -.16 & 0.58 & -.04 & -.03 & 0.22 & -.15 & 0.55 \\
BN4 & -.16 & 0.10 & 0.19 & 0.17 & 0.29 & 0.00 & -.05 & 0.07 \\
CJ4 & 0.28 & 0.03 & 0.21 & -.01 & 0.58 & -.26 & 0.07 & -.10 \\
PS4 & -.01 & 0.46 & -.17 & 0.27 & 0.17 & 0.18 & 0.24 & -.24 \\
IS4 & -.11 & 0.28 & -.28 & -.17 & -.25 & 0.03 & 0.26 & -.09 \\
SD4 & 0.22 & -.04 & 0.54 & -.02 & 0.07 & -.06 & 0.02 & 0.57 \\
SSP3 & 0.55 & -.07 & 0.32 & -.50 & -.08 & -.37 & 0.13 & 0.39 \\
LM3 & 0.20 & 0.41 & -.12 & -.11 & 0.31 & -.34 & 0.42 & -.07 \\
DS3 & 0.26 & -.27 & 0.53 & -.24 & -.26 & -.19 & -.14 & 0.56 \\
BN3 & 0.31 & 0.06 & 0.28 & -.10 & 0.02 & 0.09 & 0.02 & 0.43 \\
CJ3 & 0.32 & -.05 & 0.25 & -.21 & 0.14 & -.26 & 0.10 & 0.43 \\
PS3 & 0.00 & 0.38 & -.02 & 0.15 & 0.01 & 0.29 & 0.36 & 0.04 \\
IS3 & 0.22 & 0.29 & 0.05 & -.30 & -.08 & 0.12 & 0.31 & 0.26 \\
SD3 & 0.26 & -.11 & 0.48 & -.12 & -.11 & -.03 & -.01 & 0.64 \\
SSP2 & 0.10 & -.21 & 0.16 & -.39 & -.13 & -.19 & -.07 & 0.36 \\
LM2 & 0.49 & 0.18 & 0.14 & -.02 & 0.34 & -.28 & 0.32 & 0.33 \\
DS2 & 0.60 & -.22 & 0.57 & -.25 & -.07 & -.17 & 0.18 & 0.71 \\
BN2 & 0.35 & 0.14 & 0.37 & -.11 & -.01 & 0.04 & 0.12 & 0.56 \\
CJ2 & 0.18 & -.25 & 0.26 & 0.03 & 0.45 & 0.04 & -.24 & 0.28 \\
PS2 & 0.25 & 0.09 & 0.41 & -.12 & -.04 & 0.47 & -.11 & 0.35 \\
IS2 & 0.38 & 0.17 & 0.18 & -.16 & 0.10 & -.02 & 0.29 & 0.35 \\
SD2 & 0.32 & -.20 & 0.51 & -.17 & -.10 & -.02 & -.08 & 0.68 \\
\hline
\end{tabular}

